\title{
O estudo dos componentes do ciclo hidrológico desde métodos tradicionais até o uso de sensoriamento remoto: uma revisão
}

\author{
BRITTO, Marcela ${ }^{1}$ \\ BAPTISTA, Gustavo M. de Mello ${ }^{2}$ \\ LIMA, Erondina Azevedo de ${ }^{3}$ \\ 11nstituto de Geociências, Universidade de Brasília, Brasília, Brasil. marceladbritto@gmail.com \\ 2Instituto de Geociências, Universidade de Brasília, Brasília, Brasil. gmbaptista@gmail.com \\ 3Instituto de Física, Universidade de Brasília, Brasília, Brasil. erondinaazevedo@gmail.com
}

\begin{abstract}
Resumo
O crescimento populacional acelerado acarreta a ampliação de ambientes urbanos, trazendo impactos ambientais como a supressão da vegetação e aumento de áreas impermeáveis, os quais afetam diretamente a qualidade e disponibilidade da água. Sendo assim, é importante para o planejamento urbano a pesquisa e o levantamento de informações sobre os componentes do ciclo hidrológico nas cidades, a fim de possibilitar a tomada de decisão e estabelecer medidas mitigadoras de impactos presentes e futuros. Os componentes do ciclo hidrológico se dividem basicamente em áreas de interceptação da água da chuva, áreas de infiltração e áreas de escoamento superficial. Cada um deles desempenha uma função específica no ciclo da água em determinada região. Os pesquisadores se utilizam de técnicas clássicas/tradicionais ou mais recentes a depender do objetivo da pesquisa a ser desenvolvida. O presente artigo reúne e apresenta informações relevantes a respeito dos métodos utilizados para a pesquisa dessas áreas e traz o sensoriamento remoto como ferramenta útil, a qual tem a capacidade de levantar informações de forma rápida e eficaz, como alternativa de mapeamento e monitoramento dos elementos do ciclo hidrológico em ambientes urbanos.
\end{abstract}

Palavras-chave: componentes do ciclo hidrológico; sensoriamento remoto; interceptação; infiltração; escoamento superficial.

\begin{abstract}
The expansion of urban areas results in environmental impacts such as suppression of vegetation and increase of impermeable areas, which directly affect the quality and availability of water supply. Thus, it is important for urban planning activities to research and gather information about the elements of the hydrological cycle in urban areas, to understand its importance in the water cycle in order to establish mitigating measures for present and future impacts. The components of the cycle are basically rainwater interception areas, infiltration areas and surface runoff areas. Each one of those has a specific function in the water cycle. Researchers use classic/traditional or more recent techniques depending on the purpose of the research. This article gathers and presents relevant information regarding the methods used in this field and brings remote sensing as a useful tool, which has the capability to gather information quickly and efficiently, as an alternative to mapping and monitoring the elements of the hydrological cycle in urban environments.
\end{abstract}

Key-Words: components of the hydrological cycle; remote sensing; interception; infiltration; surface runoff. 


\section{Introdução}

O crescimento populacional e o avanço da urbanização em ambientes naturais têm acarretado impactos ambientais de diferentes naturezas (ALVES, 2015). A impermeabilização pode causar enchentes e a diminuição da qualidade da água. (THOMAS; HENDRIX; CONGALTON, 2003).

Paz (2004) exemplifica que a interceptação da água da chuva pela vegetação que ocorre em áreas de mata nativa é muito superior à de áreas agrícolas. Por conseguinte, ao analisar impactos decorrentes da ocupação humana é importante conhecer a presença e distribuição espacial dos componentes do ciclo hidrológico, visando obter resultados que traduzam a interferência da modificação da paisagem no seu funcionamento natural.

O ciclo da água na terra é decorrente da influência da radiação solar, que faz com que seja evaporada a água dos oceanos e da superfície terrestre (TUCCI; CLARKE, 1997). Os mesmos autores afirmam que "essa água entra no sistema de circulação geral da atmosfera, que depende das diferenças de absorção de energia (transformação em calor) e da reflectância entre os trópicos e das regiões de maior latitude, como as regiões polares (TUCCl; CLARKE, 1997)."

A vegetação tem um papel de importância nas trocas de energia entre atmosfera e superfície e consequentemente nos fluxos de volume de água, visto que a parcela inicial de precipitação é retida pela superfície da vegetação (interceptação) e, posteriormente, esse volume retido é evaporado. Quando o volume total de água retido no solo é evaporado, a planta começa a perder umidade pela transpiração e se inicia a necessidade de retirar água do solo através das raízes (TUCCI; CLARKE, 1997).

Análises do desenvolvimento urbano com a utilização de imagens de satélite, constituem-se numa ferramenta de extrema importância, isto porque permitem identificar possíveis vetores norteadores da dinâmica de ocupação e mudanças na paisagem (BIAS, 2003).

Um dos principais objetivos do sensoriamento remoto é o de distinguir entre as composições de diferentes materiais superficiais por meio de respostas espectrais e identificar diferentes tipos de vegetação, padrões de uso do solo, solo exposto, corpos hídricos, rochas e outros. Essa distinção é possível devido ao fato de os materiais superficiais terem comportamentos específicos ao longo do espectro eletromagnético, comportamentos esses que podem, portanto, serem usados para identificálos (CRÓSTA, 1992).

Para obter informações sobre a cobertura de áreas extensas, de maneira relativamente rápida, precisa e econômica, o sensoriamento remoto, é uma importante ferramenta no mapeamento, identificação e monitoramento de feições terrestres permitindo análises das mudanças ocorridas no uso do solo (SOUSA et. al., 2009).

Visto isso, o presente artigo de revisão tem o objetivo de apresentar o estado da arte e exemplificar alguns métodos tradicionais de estudo dos componentes do ciclo hidrológico (interceptação, infiltração e escoamento superficial), bem como evidenciar a sua importância para a compreensão do balanço hídrico em áreas urbanas. Visa, ainda, discutir a utilização do sensoriamento remoto como ferramenta para identificação e mapeamento dos componentes do ciclo por meio de técnicas de classificação e índices espectrais.

\section{Desenvolvimento}

\subsection{Interceptação da água da chuva}




\subsubsection{Fatores que influenciam na interceptação da água da chuva}

Interceptação da água da chuva é um processo físico protagonizado pela vegetação de qualquer ambiente (urbano ou florestal), em que a precipitação é interceptada, fracionada e distribuída pelo dossel, estratos inferiores, tronco e serrapilheira. Parte da água que fica armazenada na massa vegetal é evaporada para a atmosfera e o remanescente desce pelo tronco atingindo a serrapilheira e o solo (CALUX; THOMAZ, 2013).

A interceptação da chuva obtida pela cobertura vegetal tem fundamental participação nos processos hidrológicos, geomorfológicos e pedológicos do sistema vertente. Em decorrência da interceptação há o retardamento do processo de escoamento superficial, diminuição da força cinética das gotas de chuva, protegendo o solo contra o efeito de salpico. Influencia, ainda, a dinâmica do balanço hídrico, portanto a vegetação exerce importante função dentro do sistema hidrológico, visto que é um elemento regulador e armazenador da precipitação recebida, principalmente por meio da interceptação (THOMAZ, 2005 apud CALUX; THOMAZ, 2013).

Esse processo desempenha função importante para a manutenção do fluxo hidrológico de bacias urbanas, uma vez que reduz o impacto do montante de água pela barreira do dossel, o que impacta diretamente a também redução de picos de vazão do sistema de drenagem, dado a diminuição da quantidade de água que chega em sistemas de esgoto e leitos de rios e lagos urbanos (LIVESLEY; BAUDINETTE; GLOVER, 2014).

Além disso, a arquitetura das árvores e as propriedades dos troncos influenciam grandemente a proporção de chuvas interceptadas que se transformam no stemflow (fluxo do tronco) e são direcionadas para a base e para os vasos radiculares. O fluxo da água pelo tronco pode ser extremamente importante para o ciclo hidrológico e para a redistribuição de nutrientes em ambientes áridos, de agricultura, de floresta e de área urbana (LIVESLEY; BAUDINETTE; GLOVER, 2014).

Destaca-se que a maioria dos estudos de interceptação são realizados em áreas de floresta, natural ou manejada, onde é possível medi-la com mais precisão. Em ambientes urbanos o espaçamento e variabilidade entre árvores dificulta a medição, mas ainda assim pode-se esperar que a interceptação seja maior em ambientes florestais devido à cobertura de dossel contínuo (LIVESLEY; BAUDINETTE; GLOVER, 2014).

A modelagem realizada em alta resolução temporal, contribui para investigar como a interceptação influencia no tempo de concentração da bacia hidrográfica, um parâmetro importante no controle de inundações (XIAO et al., 2000). Nesse sentido, entender como diferentes características do dossel das árvores dos traços foliares e propriedades do caule influenciam o input de precipitação, distribuição e escoamento contribuem para a modelagem da hidrologia urbana (LIVESLEY; BAUDINETTE; GLOVER, 2014).

\subsubsection{Dados coletados in-situ}

A quantificação da interceptação da água da chuva se dá pela diferença entre a precipitação total e a quantidade de água que passa pelo dossel, sendo este processo influenciado pelo tipo de evento de chuva; espécie da vegetação e estrutura do dossel; e clima da região (LIVESLEY; BAUDINETTE; GLOVER, 2014).

Os autores Livesley, Baudinette e Glover (2014) realizaram um estudo para testar a aplicabilidade dos métodos de campo que se utilizam de calhas (abaixo do dossel e na base do tronco) para cálculo de interceptação, com base em 3 hipóteses:

1. Uma árvore isolada com área maior índice de área da planta leva a maior interceptação; 
2. A porcentagem de água da chuva interceptada pode decair com o aumento da magnitude do evento de precipitação;

3. Uma árvore isolada com o caule liso teria maior fluxo pelo caule do que uma árvore com caule áspero, caso tivessem arquitetura e forma de ramificação similares.

A interceptação do dossel e o fluxo do tronco de uma E. saligna madura de tronco liso e de uma árvore de E. Nicholii de tronco áspero, foram medidas de forma contínua por cinco meses no campus Barnley da Universidade de Melbourne, Victoria, Austrália. (LIVESLEY; BAUDINETTE; GLOVER, 2014).

O estudo demonstra que árvores com maior índice de área foliar (densidade de dossel) interceptaram uma quantidade maior de precipitação bruta. Para um ano típico de chuvas, isso equivale a uma redução de $45 \%$ na precipitação que chega à superfície do solo (LIVESLEY; BAUDINETTE; GLOVER, 2014).

\subsubsection{Alternativas de modelos para representar o processo de interceptação}

O melhor entendimento das perdas por interceptação pode levar a melhoria do manejo de vegetação em ambientes urbanos. Os autores Xiao et al. (2000) desenvolveram modelo tridimensional estocástico e de base física para previsão de interceptação por árvores urbanas. O modelo leva em consideração a precipitação, dados meteorológicos e a arquitetura dos dosséis como variáveis explícitas importantes (XIAO et al., 2000).

As diferenças entre características dos dosséis das árvores, estrutura foliar e propriedades do caule influenciam o input de precipitação, sua distribuição e escoamento superficial e são de grande ajuda para a modelagem hidrológica do sistema urbano de captação de água (XIAO et al., 2000).

Os referidos autores pontuam que há diferença significativa nos processos de escoamento pelo tronco e de gotejamento pelo dossel quando se observa um único indivíduo arbóreo e quando se observa todo um dossel. A Figura 1 exemplifica o processo de interceptação nos dois casos levando em consideração o ângulo do dossel $(\varphi \cdot)$, a precipitação $(P)$, a evaporação $(E)$, a água armazenada no dossel (C), a precipitação que atravessa diretamente a superfície foliar (TH), o fluxo de tronco (ST) e o gotejamento do dossel que atinge o solo (D).

Figura 1 - Modelo representativo da interceptação da água da chuva

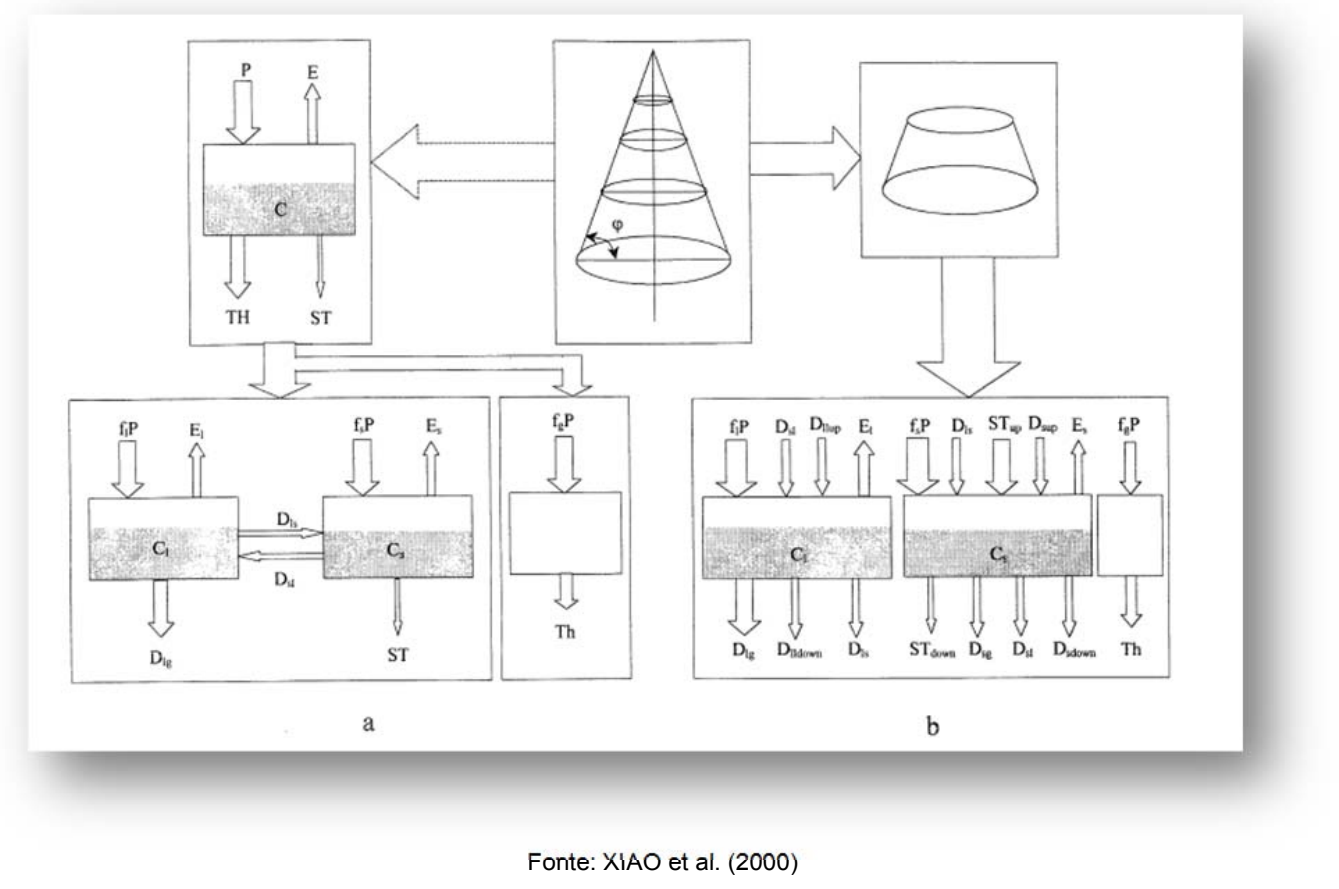


Assumindo que não há absorção de água na superfície da árvore, a precipitação que atinge o dossel é fracionada e parte é interceptada pelas folhas (fIP), parte pelos galhos (fsP) e parte passa direto pelas lacunas e atinge o solo (fgP). Todos esses fatores são influenciados pelas características da planta, por variações sazonais (XIAO et al., 2000) e/ou antrópicas e interferem no balanço hídrico local.

Ao analisar duas espécies distintas de árvores (Pyrus calleryana e Quercus suber), o estudo pôde demonstrar que a capacidade de estoque do dossel teve maior influência na interceptação e que as perdas de interceptação são mais sensíveis à área de superfície foliar (XIAO et al., 2000).

Uma desvantagem desse tipo de modelagem é que são necessários dados horários de precipitação para captar informações de chuvas rápidas e leves e as diferenças de intensidades, os quais são pontos críticos para previsão da redistribuição da chuva e do escoamento superficial (XIAO et al., 2000).

Ao analisar os resultados deve-se considerar os aspectos positivos (contenção da água da chuva) e os negativos (redução da água de recarga) da influência dos dosséis. Os benefícios são otimizados quando o dossel cobre áreas impermeáveis e os negativos aparecem quando o dossel cobre espaços verdes. $O$ estudo conclui que o fluxo do tronco foi menor para o tronco áspero ( $E$. nicholii) quando comparado com o tronco liso (E. saligna). No entanto, as estimativas anuais de fluxo de caule indicam que, mesmo para a E. saligna, o fluxo até a superfície do solo compensaria apenas aproximadamente $10 \mathrm{~mm}$ dos $200 \mathrm{~mm}$ interceptados pelo dossel (LIVESLEY; BAUDINETTE; GLOVER, 2014).

Nesse contexto, são necessárias ferramentas de gestão que analisem como o aumento da floresta e da área permeável adjacente em meio urbano pode resultar na naturalização do ciclo hidrológico. Conceitualmente, a cobertura florestal aumenta a interceptação, os solos permeáveis e a atividade da raiz das árvores aumentam a infiltração, e a floresta e os solos permeáveis combinados aumentam a evapotranspiração (WANG; ENDRENY; NOWAK, 2008).

Em resumo, o importante papel que o fracionamento da precipitação pela vegetação pode desempenhar no balanço hídrico e, portanto, nos recursos hídricos é notório. Esse fracionamento implica na redistribuição da chuva pela vegetação, como a água que passa através do dossel, afeta o balanço hídrico em escala local e também de captação. Sendo assim, a cobertura vegetal pode ter efeitos significativos na hidrologia (LLORENS; DOMINGO, 2007).

Apesar de ser ainda mais relevante em áreas onde a água é um fator limitante do desenvolvimento econômico (LLORENS; DOMINGO, 2007), mais recentemente diversos locais com anterior abundância de água estão hoje sofrendo com a escassez devido à falta de gestão adequada tanto da vegetação, quanto dos recursos hídricos em si.

\subsection{INFILTRAÇÃO e ESCOAMENTO SUPERFICIAL}

\subsubsection{Fatores que influenciam a infiltração e o escoamento superficial}

Ao atingir o solo a água da chuva pode infiltrar ou escoar superficialmente, a depender do tipo e uso do solo naquele local. A interface solo-vegetação-atmosfera exerce forte influência sob o ciclo hidrológico e, associado aos processos ecossistêmicos já naturalmente complexos, se insere nesse contexto também a ação humana (TUCCI; CLARKE, 1997).

Alterações da vegetação interferem nas propriedades do solo, refletindo nas propriedades da água dos rios, ou seja, sua presença ou ausência pode influenciar nas características da água e no ciclo hidrológico, entendimento de fundamental importância para a sustentabilidade do ambiente. Com o objetivo e preocupação atual e recorrente de preservação desses recursos, surge a necessidade de 
análise das nuances específicas de cada ecossistema e análise dos principais componentes do ciclo hidrológico (BALBINOT ET. AL, 2008).

A urbanização aumenta a quantidade de áreas impermeáveis e, assim, minimiza a infiltração e fluxo subsuperficial na área de bacias hidrográficas (CHUI; TRINH, 2016). A infiltração no solo é um importante parâmetro do movimento da água, que afeta a quantidade de escoamento e o suprimento de água no solo (YANG; ZHANG, 2011).

Além das áreas construídas e pavimentadas, outras superfícies permeáveis são também frequentemente compactadas, o que torna a superfície lisa e menos porosa, prejudicando o efeito de desvio para a subsuperfície. A compactação excessiva do solo decompõe os agregados e reduz o conteúdo de água do solo devido à redução da porosidade do ar e dos capilares. Tais alterações nas características do solo afetam sua função ecológica e acabam resultando em maiores riscos de inundação e degradação, pois acarretam consequências como o aumento do pico de vazão e a uma diminuição do fluxo de base dos corpos hídricos. (YANG; ZHANG, 2008. CHUI; TRINH, 2016).

Rahardjo et al. (2004) citado por CUOMO; DELLA SALA (2013) evidenciaram que o tempo de escoamento diminui com o aumento da intensidade da chuva e a taxa de infiltração diminui com o aumento da duração do evento de chuva. Os dados coletados sugerem que cerca de $40-74 \%$ da quantidade total de precipitação contribui para a infiltração, dependendo da intensidade da chuva, duração e condições de umidade antecedentes na inclinação.

Os parâmetros são correlacionáveis e mostram que a infiltração e o escoamento superficial são processos dependentes entre si e que os fatores específicos de cada ambiente influenciam os dois fenômenos de maneira significativa (CUOMO; DELLA SALA (2013).

\subsubsection{Métodos de investigação do comportamento da infiltração e do escoamento superficial}

Os autores Cuomo, Della e Sala (2013) afirmam que uma estimativa adequada da descarga de escoamento requer necessariamente a avaliação da quantidade de precipitação que se infiltra na superfície do solo. Na literatura, existem diferentes métodos de modelagem que podem ser divididos em dois grupos principais: empírico e físico (CUOMO; DELLA SALA, 2013).

O método Curva-Número, por exemplo, é um método empírico bem conhecido e é baseado em uma equação de balanço de massa entre a precipitação acumulada desde o início da chuva, o escoamento ocorrido e as perdas iniciais de água antes da geração de escoamento (CUOMO; DELLA SALA, 2013).

Já o método de Green-Ampt (GA) é um método de base física que considera a infiltração vertical unidimensional baseado na lei de Darcy. O método pressupõe a presença de uma fina camada contínua de água na superfície do solo provoca uma frente de escoamento que se move para baixo em um solo homogêneo com um teor inicial uniforme de água (CUOMO; DELLA SALA, 2013). A Lei de Darcy basicamente traduz a resposta de um aquífero ao bombeamento por meio de um poço, explica o fluxo de um fluido através de um meio poroso (CABRAL, 2000).

Ambas as classes de métodos têm limitações importantes. Por exemplo, o método $\mathrm{CN}$ não considera explicitamente as propriedades hidráulicas de um solo insaturado/saturado enquanto investiga o efeito do ângulo de inclinação; por outro lado, o método modificado de Green-Ampt (MEIN e LARSON, 1973) permite considerar propriedades de solo mensuráveis como a condutividade hidráulica saturada do solo e o teor de umidade inicial, mas se refere a um padrão de infiltração vertical unidimensional que não é o caso geral infiltração de água em um declive. Portanto, diferentes simplificações evitam que ambos os métodos simulem adequadamente os processos de infiltração e escoamento (CUOMO; DELLA SALA, 2013).

Em geral, a dispersão de dados é um ponto fraco deste tipo de abordagem, especialmente para 
propósitos de projeções e está principalmente relacionado a: condições in situ variáveis; diferentes resoluções temporais dos dados; e diferentes mecanismos que governam os movimentos de massa do solo (CUOMO; DELLA SALA, 2013).

Yang e Zhang (2011) realizaram em Nanjing (China) um estudo se utilizando de trinta locais representativos que pertencem a quatro tipos de zonas funcionais urbanas, definidos como uma área especial para atividades humanas, tais como áreas residenciais, áreas de parque, cinturão verde e estradas. Esses locais foram reagrupados em dez padrões de acordo com zonas funcionais urbanas, tipos de vegetação e tempo de uso da terra. Foram colhidas três amostras de solo de cada sítio a ser analisado. Visto que a compactação do solo ocorre na camada superficial, as amostras foram coletadas em uma profundidade de até $10 \mathrm{~cm}$ (YANG; ZHANG, 2011).

A taxa inicial de infiltração foi taxa obtida no primeiro minuto de avaliação. Alternativamente, a taxa final de infiltração foi registrada quando a taxa de infiltração se estabilizou ao longo do tempo (YANG; ZHANG, 2011).

Geralmente, o escoamento superficial ocorre apenas quando a intensidade da chuva é maior que a capacidade de infiltração. O coeficiente de escoamento foi calculado de acordo com as equações, em que Ri é a profundidade do escoamento superficial $(\mathrm{mm})$, Ki é a intensidade do evento de chuva (com intensidade maior do que a capacidade de infiltração), Ks é a infiltração final, t é a duração do evento de chuva (h), ai é o coeficiente de escoamento superficial (\%) e Pi a precipitação total (mm) (YANG; ZHANG, 2011):

$$
\begin{aligned}
& R_{i}=\left(K_{i}-\mathrm{Ks}\right) \times t \\
& a_{i}=\frac{R_{i}}{P_{i}} \times 100
\end{aligned}
$$

Coeficiente de Pearson foi utilizado para obter a correlação entre as taxas de infiltração e outras propriedades físicas e químicas do solo. As taxas de infiltração inicial e final foram correlacionadas com a densidade do solo, porosidade total, porosidade preenchida (com ar), porosidade capilar e teor de matéria orgânica, areia, silte e argila (YANG; ZHANG, 2011). Grandes variações nas taxas finais de infiltração foram observadas em padrões idênticos de uso da terra, porém a taxa de infiltração do solo sempre diminuiu com o aumento da densidade e diminuição da porosidade (YANG; ZHANG, 2011).

Gráfico 1 - Comparativo entre níveis de compactação do solo e taxa de infiltração final

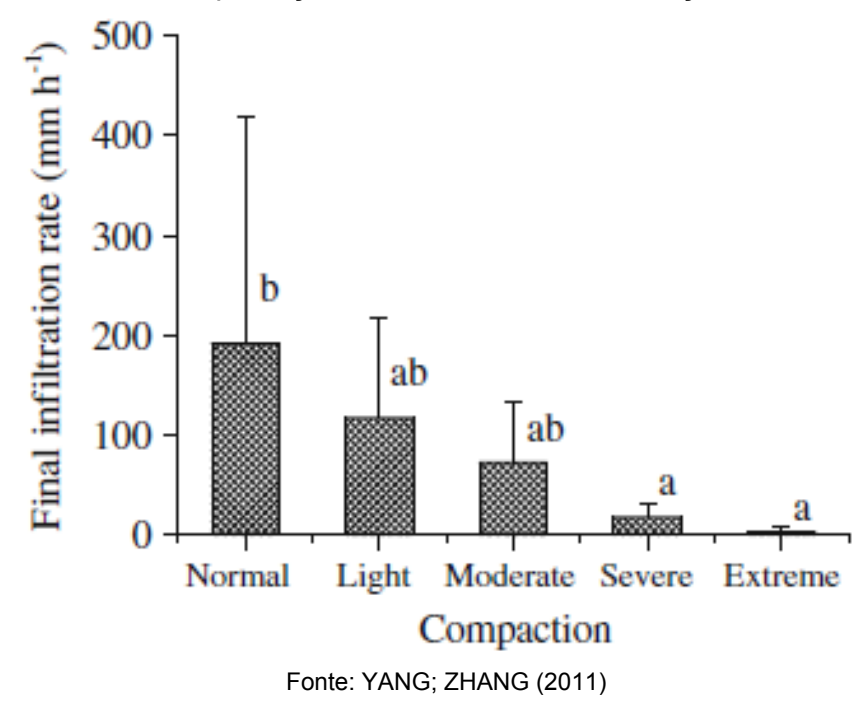


O Gráfico 1 acima ilustra a comparação entre taxa de infiltração final e a compactação do solo. É possível notar que a taxa final de infiltração diminuiu com o aumento do grau de compactação do solo. Além disso, a taxa final de infiltração do solo não compactado (normal) foi significativamente diferente daquela dos solos severamente e extremamente compactados (YANG; ZHANG, 2011).

Os resultados mostraram que a taxa de infiltração diminui com o aumento da densidade superficial do solo e consequente redução da sua porosidade, chegando a conclusão de que a presença de macro poros aumenta a taxa de infiltração e reduz o escoamento superficial (YANG; ZHANG, 2011).

Os autores Yang e Zhang (2011) explicam que no início da infiltração, o solo está seco e a água se move rapidamente pela gravidade e sucção da matriz. No entanto, à medida que a infiltração avança, a força da sucção da matriz diminui gradualmente e a umidade do solo aumenta. Como resultado, a taxa de infiltração diminui gradualmente. Quando a sucção da matriz é próxima de zero, a água se move apenas por gravidade e a taxa de infiltração se estabiliza com o tempo (YANG; ZHANG, 2011).

De maneira complementar, Zhang, Jiao e Yang (2000) observaram que a taxa de infiltração depende do teor de água inicial do solo e que a presença de descontinuidades estratigráficas pode influenciar o padrão de infiltração, em estudo realizado na província de Hubei (China) (ZHANG; JIAO; YANG, 2000).

Além disso, a qualidade da água de escoamento superficial foi baixa durante os eventos de inundação estudados. Portanto, a baixa taxa de infiltração, devido à compactação do solo, afeta negativamente o ambiente ecológico urbano, aumenta a taxa de enchentes instantâneas e degrada a qualidade da água superficial (YANG; ZHANG, 2011).

A fim de mitigar as mudanças nos regimes hidrológicos em áreas urbanas, uma nova abordagem de manejo de água pluviais, chamada de desenvolvimento de baixo impacto, vem sendo adotada nas últimas décadas (CHUI; TRINH, 2016).

A ideia geral é implementar controles hidrológicos descentralizados em pequena escala ao longo do território de uma bacia para replicar o regime hidrológico. Os gramados urbanos são um exemplo de unidade de controle hidrológico que foca em aumentar a capacidade de infiltração (CHUI; TRINH, 2016) e é o mais comum de se ver em áreas urbanizadas.

No contexto de áreas urbanas, é interessante ressaltar que a cobertura de grama reduz significativamente o escoamento, sendo necessária uma pequena modificação da estrutura micro topográfica para verificar um efeito significativo no processo de escoamento superficial (DARBOUX et al., 2002. PAN; SHANGGUAN, 2006).

Essas estruturas baseadas em infiltração, reintroduzem o "desvio" da subsuperfície e, portanto, reduzem o escoamento superficial e influenciam a recarga do armazenamento subterrâneo, o que contribui para a manutenção dos fluxos de base (CHUI; TRINH, 2016).

Para entender melhor os processos de infiltração transitória e mais especificamente as taxas de infiltração em diferentes cenários, simulações de infiltração unidimensionais foram realizadas por Chui e Trinh (2016) utilizando um solucionador genérico de equações diferenciais parciais em Cingapura, por meio de um estudo de caso da bacia de Marina para examinar a infiltração em grande escala e a viabilidade de restaurar a infiltração com um planejamento em nível de captação usando simulação numérica. $\mathrm{O}$ estudo de caso adota modelo digital de elevação (MDE), cobertura do solo e mapa do solo. O modelo é calibrado usando o nível de água perto da saída da captação, medido pela agência nacional de água de Cingapura em abril de 2014 (CHUI; TRINH, 2016).

As taxas de infiltração são calculadas em diferentes cenários representando várias condições: 
intensidade de chuva constante, diferentes propriedades do solo e eventos de chuva representativos (CHUI; TRINH, 2016).

Foi possível observar que o volume de infiltração é maior para chuvas com intensidade mais alta e constante. No entanto, se forem considerados eventos pluviométricos com as mesmas profundidades de precipitação, a fração de precipitação que infiltra é, na verdade, menor para os de maior intensidade e menor duração (CHUI; TRINH, 2016).

Esses processos confirmam os resultados da análise de dados de campo e simulações em escala local baseadas em eventos de chuva. Por exemplo, a taxa de infiltração é influenciada pela condutividade hidráulica e intensidade da chuva, conteúdo de umidade do solo e o intervalo de tempo entre os eventos de precipitação. (CHUI; TRINH, 2016).

Bryan e Rockwell (1998), em estudo realizado em Toronto se utilizando de amostras de solo de composição areia/argila, mostraram que o tempo médio de escoamento (tempo decorrido entre o início da chuva e a geração de escoamento) diminui quando os ângulos de inclinação aumentam.

A taxa de infiltração aumenta com a inclinação e intensidade da chuva. Em particular, este efeito é mais pronunciado para maior valor da intensidade da chuva. Além disso, à medida que a intensidade da chuva aumenta, o tempo de escoamento é mais curto, a curva de infiltração diminui mais rapidamente e a taxa de infiltração estável após uma longa exposição à chuva torna-se maior, concluem Assouline e Ben-Hur (2006).

\subsection{SENSORIAMENTO REMOTO}

\subsubsection{Histórico das imagens de sensoriamento remoto}

O termo sensoriamento remoto foi cunhado em 1960 por Pruit e colaboradores. É uma tecnologia altamente bem-sucedida para coleta automática de dados de monitoração dos recursos terrestres em escala global. A resolução espectral de imagens obtidas por sensoriamento remoto hoje já ultrapassa centenas de bandas, o que possibilita sua aplicação nas mais diversas áreas como monitoração ambiental, detecção de desastres, defesa, vigilância etc (MENESES, 2012).

Avanços notáveis ocorreram nas últimas décadas na tecnologia de sensores, que resultaram em espectrorradiômetros capazes de adquirir medidas em campo ou laboratório com alta resolução, e o mesmo vem acontecendo com sensores a bordo de aeronaves (GALVÃO, 2019). A resposta espectral dos materiais pode ser medida a partir do espaço com sensores imageadores a bordo de satélites.

Há algumas formas de obtenção de imagens a partir da resposta espectral da Terra. Uma delas é a utilização de sensores multiespectrais que possuem um pequeno número de bandas largas (dezenas a centenas de $\mathrm{nm}$ de largura) estrategicamente posicionadas em janelas atmosféricas ao longo do espectro eletromagnético; a outra é a utilização de sensores hiperespectrais, os quais têm um grande número de bandas estreitas (>15 nm de largura), posicionadas sequencialmente em intervalos espectrais regulares, ao longo de uma determinada faixa espectral (MENESES, 2012). 
Figura 2 - Representação do modelo hiperespectral

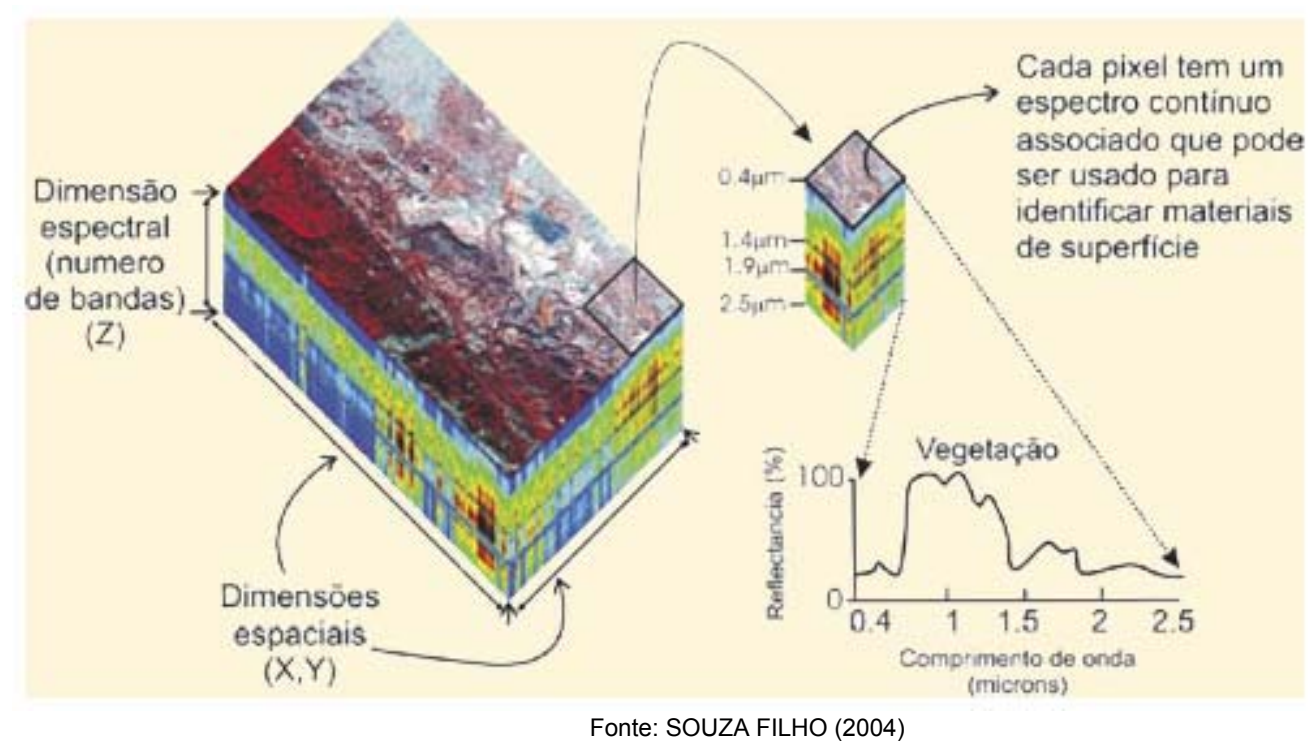

Para exemplificar o conceito de sensoriamento remoto hiperespectral pode-se utilizar um cubo de dimensões $x, y$ e $z$, sendo $x$ e $y$ as linhas e colunas da imagem e $z$ a representação das bandas do sensor de acordo com os seus comprimentos de onda (GALVÃO, 2019).

Na Figura 2 observa-se os espectros de reflectância que ilustram o comportamento de vários materiais presentes numa área urbana. O gráfico mostra o comportamento espectral da vegetação para um comprimento de onda específico. Comportamentos espectrais de outros materiais podem ser similares e distintos ao longo do espectro eletromagnético - ou seja, cada material tem uma assinatura espectral característica, que serve para distingui-los e caracterizá-los (SOUZA FILHO, 2004).

Comparado ao sensoriamento remoto multiespectral, o hiperespectral permite reconhecer nas cenas, por exemplo, componentes bioquímicos da vegetação, como a clorofila, água foliar, lignina e celulose (GALVÃO, 2019) o que permite a diferenciação entre espectros de vegetação sadia e espectros de vegetação senil.

Imagens com grande resolução espacial são úteis quando o objetivo é estudar o local em detalhe e imagens com pequena resolução espacial são melhores ao estudar áreas maiores, em escala regional, por exemplo. A identificação do alvo em imagens de sensoriamento remoto é determinada por quatro diferentes medições: 1) Resolução espacial - campo de visada do sensor; 2) Resolução espectral comprimento de onda das bandas; 3) Resolução radiométrica - medida de radiância do alvo; e 4) Resolução temporal - data de obtenção da imagem e tempo de revisita. Todas essas formas de medição atuam em conjunto em um processo interativo e devem ser levadas em conta integradamente pelo analista de forma a se obter um entendimento completo sobre os motivos pelos quais alguns objetos são identificados nas imagens (MENESES, 2012).

\subsubsection{Uso do sensoriamento remoto para estudo da vegetação e hidrologia}

O sensoriamento remoto tem se desenvolvido rapidamente nos últimos 20 anos. O sensoriamento remoto do ciclo da água, que é a fronteira da pesquisa interdisciplinar entre sensoriamento remoto e a hidrologia, tem como objetivos: estudar a teoria, metodologia e tecnologia de aquisição de componentes hidrológicos via sensoriamento remoto; e combinação de sensoriamento remoto e aplicação de modelos hidrológicos (CUI et al., 2018). 
Pesquisas sobre o ciclo da água em escalas global e regional envolvem os elementos de hidrologia e meteorologia, incluindo precipitação, evapotranspiração, escoamento, umidade do solo e armazenamento total de água em várias resoluções espaço-temporais. Esses elementos têm efeitos profundos na sociedade humana e nos ecossistemas naturais em muitos aspectos. Tais informações podem ser extraídas, simuladas e recuperadas usando técnicas de sensoriamento remoto (HEGERL et al., 2015. TANG et al., 2016. CUl et al., 2018).

Embora os pluviômetros e os radares terrestres meçam a precipitação diretamente com precisão relativamente alta, eles sofrem limitações inerentes, como distribuição e cobertura espacial limitada. Portanto, o sensoriamento remoto via satélite é uma forma prática de obter observações abrangentes de precipitação global (CUI et al., 2018).

Diversas técnicas têm sido utilizadas com sucesso para fins de mapeamento urbano no campo do sensoriamento remoto, como metodologias tradicionais de classificação (supervisionadas e/ou não supervisionadas), índices espectrais e suas derivações, integração de sensoriamento remoto e SIG, análise de paisagem em escala múltipla e modelos conceituais da superfície do solo, os quais estão entre os trabalhos mais importantes na literatura com foco no monitoramento da expansão urbana (VILLA, 2012).

A interpretação de imagens de uma única data apresenta dificuldades para alvos que possuem marcada sazonalidade climática, pois sua reflectâncias mudam no decorrer do ano. Esse é o caso, por exemplo, dos biomas Cerrado e Caatinga. No Cerrado a distribuição da precipitação ao longo dos meses é bastante irregular: seis meses de chuva e seis meses de seca, sendo comum não haver precipitação nenhuma em julho e agosto. Um mesmo alvo pode apresentar características espectrais diferentes em imagens obtidas em datas distintas ao longo do ano (SANO; BORGES, 2019).

\subsubsection{Classificação de imagens de satélite}

No passado, a classificação de imagens automatizada se baseava quase que exclusivamente em classificar cada pixel pela intensidade de cor, negligenciando características importantes de textura, forma, localização e contexto (SHARMA; SARKAR, 1998). Imagens de alta resolução permitem o mapeamento de objetos individuais e contêm mais informação espectral. A classificação deve ser exaustiva, mutuamente exclusiva e hierarquizada. A estratégia de classificação deve ter classes (vegetação, água, solo exposto) e regras que definam como encaixar as áreas definidas em cada classe (THOMAS; HENDRIX; CONGALTON, 2003. CONGALTON; GREEN, 2008).

Thomas, Hendrix e Congalton (2003) se utilizaram da classificação de áreas divididas em 2 grupos: áreas permeáveis e áreas impermeáveis. Uma abordagem de amostragem aleatória estratificada foi escolhida para garantir que as classes de cobertura da terra que ocorrem em frequências mais baixas, especialmente as características da água, foram adequadamente amostradas. Este objetivo foi alcançado selecionando um número mínimo de pontos em cada classe de uma classificação não supervisionada das imagens geradas usando o algoritmo ISODATA.

De acordo com Martins (2012), o método de classificação ISODATA é provavelmente o mais conhecido. O método identifica padrões típicos nos níveis de cinza, os quais são classificados efetuando-se o reconhecimento de alguns parâmetros escolhidos para determinar a sua interpretação.

Nesse mesmo estudo, uma comparação das diferentes matrizes mostra que o mapeamento de dados de alta resolução usando informações puramente espectrais resultou em precisões de mapas relativamente baixas, representando uma desvantagem. No entanto, a classe "vegetação" alcançou alta precisão $(90 \%)$ usando métodos de classificação espectral. O sucesso desta classe é devido à vegetação paisagística, como campos de golfe, que são espectralmente distintos do ambiente circundante, o que acontece com alguma frequência em áreas urbanas (THOMAS; HENDRIX; 
CONGALTON, 2003).

Existem alguns tipos de confusão difíceis de mapear. O paisagismo no deserto, por exemplo, geralmente consiste em cascalho, e certos tipos de cascalho podem ser espectralmente indistinguíveis do pavimento. Para tornar a confusão ainda mais complexa, uma camada de areia carregada pelo vento pode cobrir áreas pavimentadas (THOMAS; HENDRIX; CONGALTON, 2003).

Fotos ortorretificadas e o conhecimento da área de estudo são essenciais para fazer essas distinções com base em pistas de identificação visual. A técnica de classificação mais bem-sucedida para analisar essas distinções finas foi o método de modelagem espacial usando forma, textura, proximidade e contexto para resolver a confusão espectral (THOMAS; HENDRIX; CONGALTON, 2003).

O mapeamento automatizado de imagens de alta resolução requer uma nova abordagem. Com as novas fontes de imagem, até mesmo objetos individuais podem ser compostos de muitas características espectrais diferentes. Por exemplo, uma árvore pode ter o dossel composto por diferentes pixels em uma resolução de 1m (THOMAS; HENDRIX; CONGALTON, 2003).

A segmentação de imagens permite integração de informações espectrais e contextuais, que identifica unidades de mapa homogêneo, em vez de pixels individuais (THOMAS; HENDRIX; CONGALTON, 2003).

A existência de pixel mistura é uma fonte de incerteza inevitável para estimar a perda de interceptação usando observações de sensoriamento remoto, por exemplo. Um método alternativo é usar imagens de alta resolução espacial para reduzir os efeitos de mistura de pixels (CUI; JIA; HU, 2015).

\subsubsection{Monitoramento por índices de vegetação}

A infraestrutura verde de áreas urbanas é muito importante para a manutenção da qualidade de vida nas cidades. Isso desencadeia a busca por uma ligação mais robusta entre a condição da infraestrutura verde urbana e sua capacidade de suprir os serviços ecossistêmicos. Entender a condição da infraestrutura verde em ambientes urbanos fornece informação necessária à tomada de decisão de investimentos na área (DIMITROV et al., 2018).

O sensoriamento remoto oferece oportunidade para monitoramento da variabilidade espaço-temporal usando insumos básicos como a transformação e combinação da reflectância espectral da cobertura vegetal em índices de vegetação. Os que são mais utilizados empregam a informação contida nas reflectâncias de dosséis referentes às regiões do vermelho e do infravermelho próximo (MARTINS, 2012. CUI et al., 2018).

Tais índices têm a função de realçar o comportamento espectral da vegetação, correlacionando-se com seus parâmetros biofísicos em um modelo que explicita o comportamento sazonal. Incluem também elevados graus de correlação com o vigor da vegetação verde, porcentagem de cobertura do solo e a atividade fotossintética (MARTINS, 2012).

Dentre os índices de vegetação comumente citados na literatura o Índice de Vegetação por Diferença Normalizada (NDVI) é o mais amplamente utilizado. Séries multitemporais de seus valores têm sido utilizadas em vários trabalhos para auxiliar a identificação e classificação de vegetação terrestre, caracterizar a dinâmica da vegetação, estimar precipitação, gerar alertas de secas e desenvolver modelos de previsão climática (GONÇALVES, 2008).

O NDVI expressa a diferença de reflectância entre a feição de absorção do vermelho $(660 \mathrm{~nm})$ e o aumento de albedo que ocorre nos espectros de vegetação após o início do infravermelho próximo $(800 \mathrm{~nm})$ (BAPTISTA, 2004). 
Os valores de NDVI variam de -1 a +1 , sendo que valores negativos correspondem a alvos sem cobertura vegetal e valores positivos a alvos com cobertura vegetal verde e densa. Este índice é sensível a variações de biomassa verde, conteúdo de clorofila e estresse hídrico, possuindo alta correlação com a banda espectral do vermelho (SANO et al., 2019).

O índice é muito útil no exame da vitalidade das plantas, capturando a quantidade de luz que está sendo absorvida e refletida por elas e pela disponibilidade de clorofila. Tipicamente a vegetação verde tem valores entre 0,3 e 1. Em geral, se houver muito mais radiação refletida em comprimentos de onda próximos ao infravermelho do que em comprimentos de onda visíveis, é provável que a vegetação traduzida nesse pixel seja densa (DIMITROV et al., 2018).

Os autores Dimitrov et al. (2018) realizaram um estudo em algumas áreas verdes urbanas em Karlovo (Bulgária) para apresentar uma abordagem integrada na avaliação da condição da infraestrutura verde urbana por observação in-situ e sensoriamento remoto.

A pesquisa de vegetação urbana em Karlovo (Bulgária) demonstrou que a vitalidade da vegetação captada pelo NDVI corresponde totalmente com os resultados obtidos em campo (DIMITROV et al., 2018).

Outro índice existente é o SAVI (Soil-Adjusted Vegetation Index), que é ajustado para efeitos do solo. O SAVI foi baseado na inserção de uma constante L na equação do NDVI, que corresponde a 0.5 da vegetação esparsa. Quando a influência do solo é mínima, em regiões de vegetação densa, o valor de L tende a 0 (SANO et al., 2019. HUETE, 1988).

O SAVI possui alguns índices derivados: SAVI transformado, SAVI modificado e SAVI otimizado. Há também o ARVI (Atmospherically Resistant Vegetation Index), utilizado para atenuação do espalhamento da radiação na atmosfera (SANO et al., 2019).

Villa (2012) desenvolveu um estudo de técnicas de detecção de mudanças para análises de crescimento urbano que explora índices espectrais em razão de sua simplicidade e pronto uso, bem como sua confiabilidade. A área escolhida foi a região metropolitana de Milão, Itália, e foram descritas mudanças na ocupação do solo em escala regional/local se utilizando de imagens multiespectrais (VILLA, 2012).

O autor de utiliza do SVI (Soil and Vegetation Index) para discriminar área urbana e área não urbana e as mudanças ocorridas em um período de tempo. O SVI é um índice espectral baseado na resposta espectral dos alvos no que corresponde a faixa do infravermelho de ondas curtas (SWIR) e tem demonstrado excelentes performances em discriminar superfícies impermeáveis (relacionadas a classes de características de urbanização) de superfícies não-impermeáveis em trabalhos científicos publicados anteriormente (VILLA, 2012).

A abordagem metodológica apresentada demonstrou boa capacidade de discriminação para o mapeamento de evolução de áreas urbanas usando dados de sistemas sensores capazes de mapear áreas construídas de vários tamanhos (VILLA, 2012), ou seja, em diferentes escalas (local, regional, global).

Visto a interferência dos efeitos de solo e da atmosfera nos espectros da vegetação, surgiu a necessidade de se desenvolver outro índice de vegetação que atenuasse esses efeitos. Em 2002 foi criado o EVI (Enhanced Vegetation Index), que atenua os dois efeitos simultaneamente por meio da incorporação de uma constante L e de uma banda do azul. (SANO et al., 2019. HUETE et al., 2002).

O dióxido de carbono - $\mathrm{CO} 2$ tem sua importância associada ao efeito estufa, por meio do qual é mais comumente conhecido. Entretanto, é importante também para o processo de fotossíntese e sua 
concentração tem aumentado nos últimos anos (BAPTISTA, 2004).

O CO2-flux (RAHMAN et al., 2000) é um índice que mede o sequestro florestal de carbono pela vegetação fotossinteticamente ativa. Ele é obtido por meio da integração dos índices de vegetação NDVI e PRI, dois índices espectrais do tipo "diferença normalizada" (BAPTISTA, 2004. TEOBALDO; BAPTISTA, 2015). Estudos mostram que o PRI pode ser correlacionado com as taxas de fotossíntese e o NDVI com o sequestro de carbono. Isso ocorre porque quanto maior for a atividade fotossintética, maior serão as feições de absorção nas faixas do azul e do vermelho (BAPTISTA, 2004).

O PRI é o índice de reflectância fotoquímica (Photochemical Reflectance Index) (GAMON; SERRANO; SURFUS, 1997) e é especialmente sensível a mudanças no pigmento xantofila e, portanto, à eficiência do uso da luz (KOOISTRA et al., 2008).

Como o PRI é um índice normalizado e seu valor pode, teoricamente, variar de -1 a +1 , sendo necessário transformá-lo em um termo de 'eficiência' por uma manipulação algébrica simples de adicionar 1 a cada valor PRI e dividir o resultado por 2. Esse PRI reescalonado (sPRI) tem valores em um intervalo de 0 a 1 (RAHMAN et al., 2004). A multiplicação do valor do NDVI e o valor reescalonado de $\mathrm{PRI}$ resulta no CO2-flux, conforme a fórmula a seguir:

$$
\mathrm{CO}_{2}-\mathrm{flux}=\mathrm{NDVI} \times \mathrm{SPRI}
$$

Um estudo desenvolvido por Teobaldo e Baptista (2015) foi realizado com o objetivo de determinar a correlação entre severidade de queimada e sequestro de carbono, por meio da determinação do $\mathrm{CO} 2-$ flux antes e depois da queimada e no momento da rebrota. Visto que o CO2-flux é um índice espectral para investigação de sequestro de carbono, este pode ser utilizado em ampla escala para outras determinações, como por exemplo áreas de vegetação que têm influência direta no ciclo hidrológico local (TEOBALDO; BAPTISTA, 2015).

A Figura 3 é composta por duas imagens da Universidade de Brasília (UnB) - Campus Darcy Ribeiro, Asa Norte, Brasília, DF e ilustra a utilização do CO2-flux como ferramenta de análise da vegetação em diferentes períodos do ano. À esquerda está a imagem obtida em agosto de 2018 e à direita em dezembro de 2018. Os maiores valores traduzem a maior presença de vegetação fotossinteticamente ativa em tons mais claros de cinza. Já os menores valores, ao contrário, áreas sem vegetação, como áreas pavimentadas, edificadas ou de solo exposto, em tons mais escuros de cinza.

O Cerrado, bioma onde a UnB está inserida, apresenta grande contraste sazonal entre a estação seca e a estação chuvosa. A marcante sazonalidade climática exerce forte influência na fenologia da vegetação, algumas fisionomias perdem total ou parcialmente as folhas na estação seca e ocorre a produção de novas folhas, aumento da biomassa e ativação dos processos metabólicos na estação chuvosa (RATTER ET AL., 1997. SOUZA; GURGEL; CIAMP, 2016).

Nesse contexto, é possível perceber tons de cinza mais claros predominantes na imagem tomada em dezembro, pois é um período chuvoso em que a vegetação se encontra com mais vigor. Enquanto tons mais escuros de cinza, que representam áreas construídas e impermeabilizadas, não possuem variação expressiva de uma imagem para outra. 
Figura 3 - Aplicação do CO2-flux em imagem da Universidade de Brasília

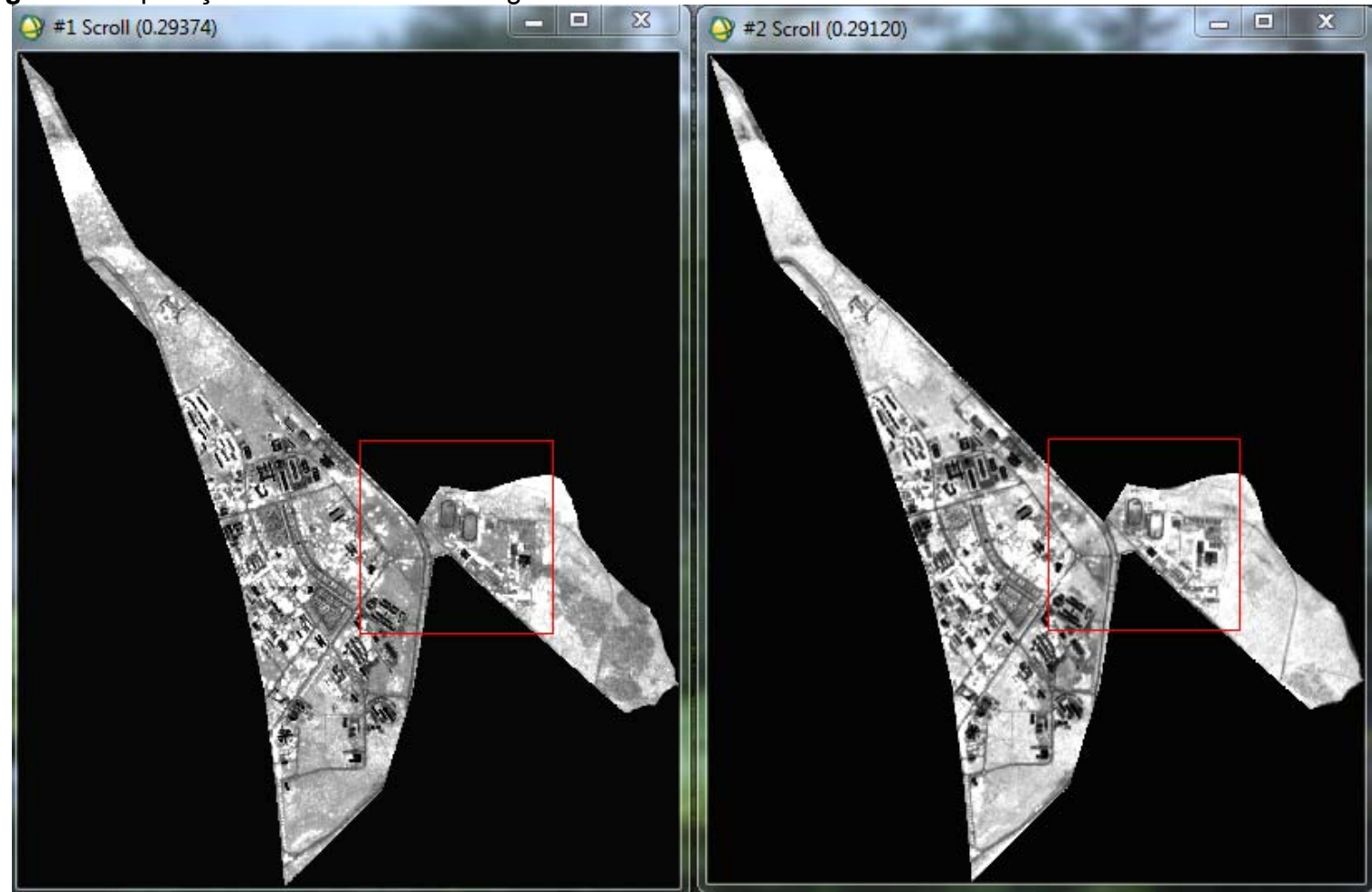

\subsubsection{Sensoriamento Remoto em áreas urbanas}

A cobertura superficial impermeável foi identificada como um indicador chave no contexto de desenvolvimento urbano e informações detalhadas sobre o tipo, densidade, configuração e conectividade de superfícies impermeáveis são necessárias para descrever as condições biológicas em uma bacia hidrográfica ou para servir como insumo para modelos ambientais (LINDEN; HOSTERT, 2009. ARNOLD; GIBBONS, 1996. ALBERTI et al., 2007).

Utilizando-se de maneira mais aprofundada do componente de área impermeável, determinar a relação do território com as áreas adjacentes, no sentido de determinar quanto da água da chuva em uma determinada superfície realmente foi transportada para um sistema de captação ou leito de corpos d'água, são informações espacialmente explícitas sobre superfícies impermeáveis e cobertura de solo urbano que servem para derivar o uso do solo como variável de entrada funcional para aplicações ecológicas urbanas (ARNOLD; GIBBONS, 1996. LINDEN; HOSTERT, 2009)

Por exemplo, em geral telhados drenam a água para jardins, gramados ou outras áreas permeáveis, então causam menos impacto que rodovias, as quais encaminham o escoamento diretamente para o sistema de captação pluvial (ARNOLD; GIBBONS, 1996).

Para medir e se utilizar da cobertura impermeável como ferramenta de proteção aos recursos hídricos é necessário saber como a impermeabilidade de um determinado local é distribuída. Para obter maior refinamento, a área impermeável pode ser detalhada por categorias de uso do solo descrevendo as respectivas funções e os impactos de cada uma no escoamento superficial e nesse sentido o sensoriamento é uma ferramenta útil para fornecer informações espacialmente explícitas em áreas urbanas (ARNOLD; GIBBONS, 1996. LINDEN; HOSTERT, 2009).

Deve-se considerar diferentes tipos de resolução temporal ao monitorar ambientes urbanos usando dados de sensoriamento remoto. Os fenômenos urbanos que progridem através de um ciclo de desenvolvimento são identificáveis, da mesma forma que a vegetação progride através de um ciclo 
fenológico. Dados atualizados de sensores remotos são chave para a maioria das aplicações urbanas (JENSEN; COWEN, 1999).

Uma descrição detalhada dos três principais elementos da heterogeneidade urbana - vegetação, estruturas construídas e materiais de superfície - é necessária para facilitar uma compreensão mais profunda das relações no sistema urbano humano natural acoplado (CADENASSO; PICKETT; SCHWARZ, 2007) e relevante para a maioria dos estudos em ambientes urbanos (LINDEN; HOSTERT, 2009)

Algumas classificações indicam intensidade de uso residencial, referindo-se à densidade de edifícios. Tal categorização, tacitamente pressupõe que todas as áreas residenciais são equivalentes. No entanto, as variações desses elementos podem influenciar o fluxo de água, nutrientes e energia (CADENASSO; PICKETT; SCHWARZ, 2007).

Os autores Cadenasso, Pickett e Schwarz (2007) apresentam uma classificação de uso e ocupação do solo que explica a natureza complexa dos sistemas urbanos. Essa classificação refina a caracterização dos componentes ecológicos da paisagem urbana por meio da divisão dos elementos em: vegetação de textura grossa (árvores e arbustos); vegetação de textura fina (gramados e gramíneas); solo exposto e pavimento; área edificada; e tipologia da área edificada.

O tipo de vegetação, o material de superfície e os edifícios influenciam na função desempenhada pelo ecossistema, devido à sua contribuição diferenciada na distribuição de materiais e energia. Os autores afirmam que destrinchar a estrutura urbana a partir das variáveis funcionais de uso e ocupação do solo pode melhorar a capacidade de testar hipóteses sobre a relação entre estrutura urbana e os serviços ecossistêmicos (funções ecológicas) fornecidos pelo meio (CADENASSO; PICKETT; SCHWARZ, 2007).

\section{Conclusão}

Para estudo da hidrologia, bem como seu monitoramento e atualização de informações temporais das modificações ocorridas no território, o sensoriamento remoto se apresenta como uma ferramenta bastante útil e com capacidade temporal e espacial satisfatórias a obtenção de dados. Por meio de imagens com diferentes variações espectrais é possível verificar o comportamento da vegetação que, como visto anteriormente, influencia diretamente no fluxo da água entre atmosfera e superfície terrestre, afetando sua qualidade e disponibilidade para usos múltiplos em ambientes urbanos.

A escolha do método de estudo está diretamente relacionada com o objetivo da pesquisa e com os resultados que se deseja obter. É muito comum e extremamente válida a combinação de mais de uma técnica para se obter resultados completos. A integração de métodos de estudo, tradicionais e medidas em campo, com dados de sensoriamento remoto é uma alternativa que serve para completar lacunas existentes em ambas as técnicas.

O artigo fornece subsídio teórico sobre a importância do conhecimento dos elementos do ciclo hidrológico para o balanço hídrico geral e disponibilidade hídrica em ambientes urbanos, apresentando as formas mais comuns de estudo dessas áreas, bem como traz o sensoriamento remoto como ferramenta alternativa para este tipo de estudo.

Os conhecimentos aqui disponibilizados podem contribuir para estudos com o objetivo de identificar, classificar e descrever a presença dos componentes do ciclo hidrológico, visto que cada um deles possui sua influência no ciclo geral da água, bem como suas modificações sazonais, por meio do processamento digital de imagens obtidas pelos mais diversos sensores orbitais disponíveis. 


\section{Referências}

ALBERTI, Marina et al. The impact of urban patterns on aquatic ecosystems: An empirical analysis in Puget lowland sub-basins. Landscape and Urban Planning, [s.l.], v. 80, n. 4, p.345-361, maio 2007. Elsevier BV. http://dx.doi.org/10.1016/j.landurbplan.2006.08.001.

ALVES, Patrícia Layne. CAPACIDADE DE INTERCEPTAÇÃO PELAS ÁRVORES E SUAS INFLUÊNCIAS NO ESCOAMENTO SUPERFICIAL URBANO. 2015. 110 f. Tese (Doutorado) - Curso de Ciências Ambientais, Estrutura e Dinâmica Ambiental, Universidade Federal de Goiás, Goiânia, 2015.

ASSOULINE, S.; BEN-HUR, M. Effects of rainfall intensity and slope gradient on the dynamics of interrill erosion during soil surface sealing. Catena, [s.I.], v. 66, n. 3, p.211-220, jun. 2006. Elsevier BV. http://dx.doi.org/10.1016/j.catena.2006.02.005.

BALBINOT, Rafaelo et al. O papel da floresta no ciclo hidrológico em bacias hidrográficas. Ambiência - Revista do Setor de Ciências Agrárias e Ambientais, Guarapuava, Pr, v. 4, n. 1, p.131-149, abr. 2008.

BAPTISTA, Gustavo Macedo de Mello. Mapeamento do Sequestro de Carbono e de Domos Urbanos de $\mathrm{CO} 2$ em Ambientes Tropicais, por meio de Sensoriamento Remoto Hiperespectral. Geografia (Rio Claro. Impresso), v. 29, p. 189-202, 2004.

BIAS, Edilson de Souza. Vantagens e Limitações das Imagens de Alta Resolução como Suporte ao Planejamento Urbano: O Exemplo de Sobradinho - Distrito Federal. 2003. 298 f. Tese (Doutorado) - Curso de Geografia, Instituto de Geociências e Ciências Exatas, Universidade Estadual Paulista, Rio Claro, 2003.

CABRAL, Jaime. Movimento das Águas Subterrâneas. In: FEITOSA, Fernando A. C.; MANOEL FILHO, João. Hidrogeologia: Conceitos e Aplicações. 2. ed. Fortaleza: Serviço Geológico do Brasil, 2000. Cap. 3. p. 35-51.

CADENASSO, Mary L.; PICKETT, Steward T. A.; SCHWARZ, Kirsten. Spatial heterogeneity in urban ecosystems: reconceptualizing land cover and a framework for classification. Frontiers in Ecology and The Environment, [s.l.], v. 5, n. 2, p.80-88, mar. 2007. Wiley. http://dx.doi.org/10.1890/15409295(2007)5[80:shiuer]2.0.c0;2.

CALUX, Jucelmo; THOMAZ, Edivaldo Lopes. INTERCEPTAÇÃO E PRECIPITAÇÃO INTERNA: COMPARAÇÃO ENTRE FLORESTA OMBRÓFILA MISTA E Pinus elliotttii var. elliotti?. Geoambiente On-line, [s.l.], n. 19, p.01-16, 3 jan. 2013. Universidade Federal de Goiás. http://dx.doi.org/10.5216/revgeoamb.v0i19.26049.

$\mathrm{CHUI}$, Ting Fong May; TRINH, Dieu Huong. Modelling infiltration enhancement in a tropical urban catchment for improved stormwater management. Hydrological Processes, [s.I.], p.4405-4419, 2016. Wiley. http://dx.doi.org/10.1002/hyp.10926.

CONGALTON, Russel G.; GREEN, Kass. Assessing the Accuracy of Remotely Sensed Data: Principles and Practices.2. ed. Boca Raton: Crc Press, 2008. 210 p.

CRÓSTA, Álvaro Penteado. Processamento Digital de Imagens de Sensoriamento Remoto. Campinas, São Paulo: Unicamp, 1992. 170 p.

CUI, Yaokui; JIA, Li; HU, Guangcheng. Mapping of Interception Loss of Vegetation in the Heihe River Basin of China Using Remote Sensing Observations. leee Geoscience And Remote Sensing Letters, [s.I.], v. 12, n. 1, p.23-27, jan. 2015. Institute of Electrical and Electronics Engineers (IEEE). http://dx.doi.org/10.1109/lgrs.2014.2324635.

CUI, Yaokui et al. Global water cycle and remote sensing big data: overview, challenge, and opportunities. Big Earth Data, [s.I.], v. 2, n. 3, p.282-297, 3 jul. 2018. Informa UK Limited. http://dx.doi.org/10.1080/20964471.2018.1548052. 
CUOMO, S.; DELLA SALA, M. Rainfall-induced infiltration, runoff and failure in steep unsaturated shallow soil deposits. Engineering Geology, [s.I.], v. 162, p.118-127, jul. 2013. Elsevier BV. http://dx.doi.org/10.1016/j.enggeo.2013.05.010.

DARBOUX, $F$ et al. Evolution of soil surface roughness and flowpath connectivity in overland flow experiments. Catena, [s.I.], v. 46, n. 2-3, p.125-139, jan. 2002. Elsevier BV. http://dx.doi.org/10.1016/s0341-8162(01)00162-x.

DIMITROV, Stelian et al. Integrated assessment of urban green infrastructure condition in Karlovo urban area by in-situ observations and remote sensing. One Ecosystem, [s.I.], v. 3, p.1-23, 21 fev. 2018. Pensoft Publishers. http://dx.doi.org/10.3897/oneeco.3.e21610.

GALVÃO, Lênio Soares. Do sensoriamento remoto multiespectral para o hiperespectral: o comportamento espectral dos materiais visto do espaço. In: MENESES, Paulo Roberto et al. Reflectância dos Materiais Terrestres. Brasília: Oficina de Textos, 2019. Cap. 8 p. 249-265.

GONÇALVES, R.R.V. Relação entre a resposta espectral da cana-de-açúcar, registrada nas imagens dos satélites AVHRR/NOAA, em São Paulo, e dados agroclimáticos, no período de 2001 a 2008.2008. 163p. Dissertação (Mestrado) - Curso de Engenharia Agrícola - Faculdade de Engenharia Agrícola, Universidade Estadual de Campinas, Campinas.

HEGERL, Gabriele C. et al. Challenges in Quantifying Changes in the Global Water Cycle. Bulletin Of The American Meteorological Society, [s.I.], v. 96, n. 7, p.1097-1115, jul. 2015. American Meteorological Society. http://dx.doi.org/10.1175/bams-d-13-00212.1.

HUETE, A.r. A soil-adjusted vegetation index (SAVI). Remote Sensing Of Environment, [s.I.], v. 25, n. 3, p.295-309, ago. 1988. Elsevier BV. http://dx.doi.org/10.1016/0034-4257(88)90106-x.

JENSEN, John R.; COWEN, Dave C. Remote Sensing of Urban/Suburban Infrastructure and SocioEconomic Attributes. Photogrammetric Engineering \& Remote Sensing, Columbia, v. 65, n. 5, p.611-622, maio 1999.

KOOISTRA, Lammert et al. Assessing and predicting biodiversity in a floodplain ecosystem: Assimilation of net primary production derived from imaging spectrometer data into a dynamic vegetation model. Remote Sensing Of Environment, [s.l.], v. 112, n. 5, p.2118-2130, maio 2008. Elsevier BV. http://dx.doi.org/10.1016/i.rse.2007.10.010.

LINDEN, S. van Der; HOSTERT, P. The influence of urban structures on impervious surface maps from airborne hyperspectral data. Remote Sensing Of Environment, [s.I.], v. 113, n. 11, p.2298-2305, 16 nov. 2009. Elsevier BV. http://dx.doi.org/10.1016/i.rse.2009.06.004.

LIVESLEY, S.j.; BAUDINETTE, B.; GLOVER, D. Rainfall interception and stemflow by eucalypt street trees - The impacts of canopy density and bark type. Urban Forestry \& Urban Greening, [s.l.], v. 13, n. 1, p.192-197, 2014. Elsevier BV. http://dx.doi.org/10.1016/j.ufug.2013.09.001.

LLORENS, Pilar; DOMINGO, Francisco. Rainfall partitioning by vegetation under Mediterranean conditions. A review of studies in Europe. Journal Of Hydrology, [s.I.], v. 335, n. 1-2, p.37-54, mar. 2007. Elsevier BV. http://dx.doi.org/10.1016/j.jhydrol.2006.10.032.

MARTINS, Éder de Sousa. Compartimentação Geomorfológica do Distrito Federal. Embrapa Cerrados, Planaltina, Distrito Federal, 2004. 57 p.

MARTINS, Nuno Miguel Nogueira. Utilização de imagens de satélite de alta resolução para a extracção de elementos em ambiente urbano. 2012. 89 f. Dissertação (Mestrado) - Curso de Engenharia Geográfica, Engenharia Geográfica, Geofísica e Energia da Faculdade de Ciências, Universidade de Lisboa, Lisboa, 2012. 
MENESES, Paulo Roberto. Princípios do Sensoriamento Remoto. In: MENESES, Paulo Roberto e ALMEIDA, Tati de. Introdução ao Processamento de Imagens de Sensoriamento Remoto: CNPq, 2012. Cap. 1. p. 1-31.

PAN, Chengzhong; SHANGGUAN, Zhouping. Runoff hydraulic characteristics and sediment generation in sloped grassplots under simulated rainfall conditions. Journal Of Hydrology, [s.I.], v. 331, n. 1-2, p.178-185, nov. 2006. Elsevier BV. http://dx.doi.org/10.1016/j.jhydrol.2006.05.011.

PAZ, Adriano Rolim da. Hidrologia Aplicada. Caxias do Sul: Disciplina Ministrada na Universidade Estadual do Rio Grande do Sul, 2004. 138 p.

RAHMAN, A. F. et al. Potential of MODIS ocean bands for estimating CO2flux from terrestrial vegetation: A novel approach. Geophysical Research Letters, [s.I.], v. 31, n. 10, p.1-4, maio 2004. American Geophysical Union (AGU). http://dx.doi.org/10.1029/2004gl019778.

ROSA, Roberto. Introdução ao Sensoriamento Remoto. Uberlândia, Minas Gerais: Editora Universidade Federal de Uberlândia, 1990. 136 p.

SANO, Edson Eyji et al. Reflectância da Vegetação. In: MENESES, Paulo Roberto et al. Reflectância dos Materiais Terrestres. Brasília: Oficina de Textos, 2019. Cap. 6. p. 189-223.

SANO, Edson Eyji, BORGES, Elaine Fiúza. Séries Temporais: comportamento espectral de imagens multidatas. In: MENESES, Paulo Roberto et al. Reflectância dos Materiais Terrestres. Brasília: Oficina de Textos, 2019. Cap. 9. p. 267-289.

SHARMA, K.m.s.; SARKAR, A. A Modified Contextual Classification Technique for Remote Sensing Data. Photogrammetric Engineering \& Remote Sensing, Kharaggpur, v. 64, n. 4, p.273-280, abr. 1998.

SOUSA, K.; ALMEIDA, C.; VIEIRA, F.; MOURA, P; NETO,J. Mapeamento do uso e ocupação do solo da Bacia do Rio Descoberto - DF utilizando imagens do satélite CBERS-2. In: XIV SIMPÓSIO BRASILEIRO DE SENSORIAMENTO REMOTO, 2009, Natal. Anais eletrônicos INPE.

SOUZA FILHO, Carlos Roberto de. Sensoriamento Remoto Hiperespectral. 2004. Disponível em: <https://mundogeo.com/blog/2004/08/23/sensoriamento-remoto-hiperespectral/>. Acesso em: 01 jun. 2019.

TANG, Guoqiang et al. Statistical and Hydrological Comparisons between TRMM and GPM Level-3 Products over a Midlatitude Basin: Is Day-1 IMERG a Good Successor for TMPA 3B42V7?. Journal Of Hydrometeorology, [s.I.], v. 17, n. 1, p.121-137, jan. 2016. American Meteorological Society. http://dx.doi.org/10.1175//hm-d-15-0059.1.

TEOBALDO, Débora; BAPTISTA, Gustavo Macedo de Mello. Quantificação da severidade das queimadas e da perda de sequestro florestal de carbono em unidades de conservação do Distrito Federal ${ }^{1}$. Revista Brasileira de Geografia Física, Brasília, v. 1, n. 9, p.250-264, dez. 2015. Disponível em: <www.ufpe.br/rbgfe>. Acesso em: 20 abr. 2019.

THOMAS, Nancy; HENDRIX, Chad; CONGALTON, Russell G.. A Comparison of Urban Mapping Methods Using High-Resolution Digital Imagery. Photogrammetric Engineering \& Remote Sensing, [s.l.], v. 69, n. 9, p.963-972, 1 set. 2003. American Society for Photogrammetry and Remote Sensing. http://dx.doi.org/10.14358/pers.69.9.963.

TUCCI, Carlos; CLARKE, Robin. Impactos das mudanças da cobertura vegetal no escoamento: revisão. Revista Brasileira de Recursos Hídricos, [s.I.], v. 2, n. 1, p.135-152, 1997. Fap UNIFESP (SciELO). http://dx.doi.org/10.21168/rbrh.v2n1.p135-152.

VILLA, Paolo. Mapping urban growth using Soil and Vegetation Index and Landsat data: The Milan (Italy) city area case study. Landscape And Urban Planning, [s.I.], v. 107, n. 3, p.245-254, set. 2012. Elsevier BV. http://dx.doi.org/10.1016/j.landurbplan.2012.06.014. 
WANG, Jun; ENDRENY, Theodore A.; NOWAK, David J. Mechanistic Simulation of Tree Effects in an Urban Water Balance Model 1. Jawra Journal of The American Water Resources Association, [s.I.], v. 44, n. 1, p.75-85, fev. 2008. Wiley. http://dx.doi.org/10.1111/j.1752-1688.2007.00139.x.

XIAO, Qingfu et al. A new approach to modeling tree rainfall interception. Journal of Geophysical Research: Atmospheres, [s.l.], v. 105, n. 23, p.29173-29188, 1 dez. 2000. American Geophysical Union (AGU). http://dx.doi.org/10.1029/2000jd900343.

YANG, Jin-ling; ZHANG, Gan-lin. Water infiltration in urban soils and its effects on the quantity and quality of runoff. Journal Of Soils And Sediments, [s.I.], v. 11, n. 5, p.751-761, 7 abr. 2011. Springer Nature. http://dx.doi.org/10.1007/s11368-011-0356-1.

ZHANG, J.; JIAO, J.j.; YANG, J. In situ rainfall infiltration studies at a hillside in Hubei Province, China. Engineering Geology, [s.I.], v. 57, n. 1-2, p.31-38, jun. 2000. Elsevier BV. http://dx.doi.org/10.1016/s0013-7952(99)00126-x. 